\title{
ELABELA acts as a protective biomarker in patients with atrial fibrillation
}

\author{
Chunhong Cui ${ }^{1,2 \#}$, Hongmei Zhou ${ }^{3 \#}$, Jin $\mathrm{Xu}^{3}$ \\ ${ }^{1}$ Zhoupu Hospital, Shanghai University of Medicine and Health Sciences, Shanghai, China; ${ }^{2}$ Basic Medical College, Shanghai University of Medicine \\ and Health Sciences, Shanghai, China; ${ }^{3}$ Department of Cardiology, Renji Hospital, School of Medicine, Shanghai Jiao Tong University, Shanghai, \\ China \\ Contributions: (I) Conception and design: All authors; (II) Administrative support: None; (III) Provision of study materials or patients: J Xu; (IV) \\ Collection and assembly of data: H Zhou; (V) Data analysis and interpretation: C Cui; (VI) Manuscript writing: All authors; (VII) Final approval of \\ manuscript: All authors. \\ \#These authors contributed equally to this work. \\ Correspondence to: Jin Xu. Department of Cardiology, Renji Hospital, School of Medicine, Shanghai Jiao Tong University, 160 Pujian Rd, Pudong \\ New District, Shanghai 200127, China. Email: glad-xujin@qq.com.
}

\begin{abstract}
Background: Atrial fibrillation (AF) is the most common type of clinical arrhythmia. An early diagnosis can be beneficial in the prevention of complications, such as heart failure (HF) and stroke. In this study, we revealed that ELABELA (ELA) acts as a protective factor in patients with arrhythmia and could serve as a prognostic marker for $\mathrm{AF}$ and its associated complication of $\mathrm{HF}$.

Methods: We tested the expression level of potential biomarkers including matrix metalloproteinase-9 (MMP-9), tissue inhibitor of metalloproteinase-1 (TIMP-1), and ELA with enzyme-linked immunosorbent assay (ELISA) in serum derived from 131 patients (patients with $\mathrm{AF}=103$; patients with paroxysmal supraventricular tachycardia $=28$ ). The impact of clinical risk factors and biomarkers on AF occurrence was evaluated using binary logistic analysis.

Results: The ELA expression level was lower in AF patients than in the negative controls $(\mathrm{P}<0.0001)$. Expression of ELA was negatively correlated with brain natriuretic peptide (BNP) expression in all the samples. In the binary logistic analysis, high levels of BNP and lower levels of ELA were significantly associated with an increased risk of $\mathrm{AF}(\mathrm{P}<0.0001)$ and could be used as prognostic markers for patients with $\mathrm{AF}$.

Conclusions: Expression of ELA showed a protective role in AF patients. The ELA level was negatively correlated with BNP levels, which has been shown to predict a high risk of HF independently and consistently. Additionally, lower levels of ELA were associated with a high risk of AF and HF in patients with arrhythmia.
\end{abstract}

Keywords: ELABELA (ELA); matrix metalloproteinase-9 (MMP-9); tissue inhibitor of metalloproteinase-1 (TIMP-1); atrial fibrillation (AF); brain natriuretic peptide (BNP)

Submitted Oct 14, 2021. Accepted for publication Nov 26, 2021.

doi: $10.21037 /$ jtd-21-1728

View this article at: https://dx.doi.org/10.21037/jtd-21-1728

\section{Introduction}

The global burden of an aging population is associated with an increased prevalence of arrhythmias (1). Currently, the treatment of arrhythmias is complicated because of adverse atrial remodeling (2). Atrial fibrillation (AF) is the most common arrhythmia that can lead to many life-threatening complications, such as heart failure (HF) and stroke (3). An early diagnosis of arrhythmia at risk of progressing into AF with these complications might improve the overall prognosis in such patients (4). Therapeutic devices, pharmacology, and ablation techniques have improved in this field; however, electrophysiology and blood biomarkers 
for the diagnosis of AF and its complications are still evolving.

Matrix metalloproteinase-9 (MMP-9) is a member of proteolytic enzymes family, and it participates in regulating the extracellular matrix under the balanced function of its inhibitor tissue inhibitor of metalloproteinase-1 (TIMP-1). It has been found that MMP-9 play an important role in atrial remodeling in the atrial fibrillation processing (5). Therefore, MMP-9 and TIMP-1 may be also contribute to AF complications. But the details need to be further investigated.

ELABELA (ELA) is a peptide hormone essential for development of the heart (6). ELA is the endogenous ligand of the apelin receptor, which has been found in zebrafish embryos and facilitate gastrulation activities (7). Its deficiency has been reported to promote cardiovascular malformations in mice (8). Furthermore, ELA has been shown to be a novel biomarker for right ventricular afterload and can reflect immediate pressure changes (9). Additionally, it can protect against pressure overload HF and angiotensin II-induced cardiac damage (10). However, the role of ELA as a biomarker in arrhythmias remains unclear.

It has been reported that, plasma ELA is lowly expressed in patients with $\mathrm{AF}$ compared to that without $\mathrm{AF}$ and may be potential risk factor in hypertension patients (11). In this study, we found that ELA could be benefit for preventing AF complications, such as heart failure (HF) and stroke. The prognostic value of previous clinical factors and new serum biomarkers were evaluated using different statistical analyses. We found that the ELA expression was low in patients with $\mathrm{AF}$ and negatively correlated with brain natriuretic peptide (BNP) level, indicating that ELA could be used as a serum biomarker for early diagnosis of AF. In conjunction with BNP and other high-risk factors of cardiac insufficiency, ELA could also be used to predict HF in patients with arrhythmia. We present the following article in accordance with the MDAR checklist (available at https://dx.doi.org/10.21037/jtd-21-1728).

\section{Methods}

\section{Study population}

Blood specimens were collected from 131 adult patients [103 patients with AF and 28 patients with paroxysmal supraventricular tachycardia (PSVT)]. The study was conducted with the approval of the Ethics Committee of
Renji Hospital, School of Medicine, Shanghai Jiao Tong University (approval No. KY2021-131-B) and in accordance with the Declaration of Helsinki (as revised in 2013). Samples were collected and analyzed after participants had provided their written informed consent. Only patients with arrhythmia were included in this study.

\section{Sample acquisition}

Blood samples were harvested form the cardiac cavity intraoperatively and samples were allowed to clot at room temperature for $30 \mathrm{~min}$. To isolate the clot, the samples were centrifuged at $2,000 \times \mathrm{g}$ for $20 \mathrm{~min}$ at $4{ }^{\circ} \mathrm{C}$. The serum was aliquot into $0.5 \mathrm{~mL}$ Eppendorf tubes and stored at $-80{ }^{\circ} \mathrm{C}$.

\section{Enzyme-linked immunosorbent assay (ELISA)}

ELISA was performed using a commercially available kit. The plates were pre-coated with an antibody. Diluted $100 \mu \mathrm{L}$ serum was added to the wells, and the plate was incubated at $37^{\circ} \mathrm{C}$ for $1 \mathrm{~h}$. The wells were then washed 3 times with a buffer solution. The detected antibody was diluted in blocking buffer and added to each well, followed by incubation at $37^{\circ} \mathrm{C}$ for 30 mins. The wells were again washed 3 times with a wash buffer. A working dilution of horseradish peroxidase (HRP) was added and the plate followed by incubation at $37{ }^{\circ} \mathrm{C}$ for 30 mins. The wells were washed for the last time with a wash buffer 3 times. A 3,3',5,5'-tetramethylbenzidine (TMB) substrate solution was then added to each well and incubated at room temperature for $30 \mathrm{~min}$. The stop solution was added to each well, and the absorbance was finally measured at $450 \mathrm{~nm}$ within 30 min of adding the stop solution.

\section{Statistical analysis}

For continuous variables, descriptive statistics were shown as median values, first and third quartiles, numbers, and percentages were used to present binary variables. The Wilcoxon signed rank test and Mann-Whitney $U$ test were used to investigate the statistical significance of the differences between participants with and without $\mathrm{AF}$ for continuous variables. The impact of clinical factors and biomarkers on $\mathrm{AF}$ occurrence was evaluated using binary logistic analysis.

Furthermore, one-way analysis of variance (ANOVA) was used to assess the different levels of targeted molecules in participants with clinical factors. Bivariate regression 
analysis with Spearman correlation analysis was performed to analyze the correlation between BNP and ELA, BNP, and TIMP-1.

All statistical analyses were performed using the software SPSS 23.0 (IBM Corp., Chicago, IL, USA), GraphPad Prism 9 (GraphPad Software, La Jolla, CA, USA), and RStudio (https://www.rstudio.com/). All tests were 2-tailed and stated in the text as follows: NS: not significant; ${ }^{*} \mathrm{P}<0.05 ;{ }^{* *} \mathrm{P}<0.01 ;{ }^{* * *} \mathrm{P}<0.001$.

\section{Results}

\section{Baseline characteristics}

We analyzed the blood serum of 131 patients. The baseline characteristics of the participants are shown in Table 1. In general, all participants had a greater burden of conventional risk factors. The median age of these participants, including 55 (41\%) women and $76(59 \%)$ men, was 65 years. As shown in Table 1, patients who developed $\mathrm{AF}$ were older, had a high body mass index (BMI) tendency, and the AF patients usually had greater left ventricular end-systolic diameter (LVDd), left ventricular end-systolic diameter (LVDs), and left atrium $(\mathrm{P}<0.05)$. Consistent with previous reports (12), left ventricular ejection fraction (LVEF) was decreased in AF patients (Table 1). Concerning the concentrations of the biomarkers at baseline, the AF patients had higher levels of neutrophil (NE), creatine kinase MB (CK-MB), urea nitrogen (BUN), creatinine, uric acid, potassium, total bilirubin (TB), direct bilirubin (DB), fibrinogen (Fbg), glycosylated hemoglobin (HbA1c), total triiodothyronine (TT3), and BNP $(\mathrm{P}<0.05)$ (Table 1).

\section{$E L A$ protects patients with arrhythmias against $A F$ and beart failure}

As described above, AF is the most common arrhythmia, and $\mathrm{HF}$ is one of the main life-threatening complications caused by AF (3). An early diagnosis helps prevent later complications and enables more opportunity for therapy. Matrix metalloproteinase-9 (MMP-9) and tissue inhibitor of metalloproteinase-1 (TIMP-1) have been reported to contribute to extracellular matrix remodeling of atria in $\mathrm{AF}$ (13). Kim et al. found that increasing ELA bioavailability in mice is beneficial for treating and preventing AF (14). Further, in search of new biomarkers for determining the prognosis of arrhythmia and AF, we tested serum biomarker levels through ELISA, including MMP-9, TIMP-1, and
ELA. The results showed that MMP-9 was highly expressed in $\mathrm{AF}$ patients, but it was not significant $(\mathrm{P}=0.4169)$. There was no difference in the TIMP-1 expression between participants with AF and those with PSVT (Figure S1), whereas expression of ELA was much lower in patients with $\mathrm{AF}$ than that in patients without $\mathrm{AF}$ (Figure 1A). The BNP is a proven diagnostic biomarker for HF, and the expression level of ELA was low in participants for whom BNP expression was higher than $100 \mathrm{pg} / \mathrm{mL}(\mathrm{P}=0.24)$ (Figure 1B). Furthermore, the level of ELA in serum was low in participants with hypertension $(\mathrm{P}=0.4)$ (Figure $1 C)$. The expression of ELA was significantly lower in AF participants (persistent AF, paroxysmal AF, and chronic AF) than in those with PVST $(\mathrm{P}<0.0001)$ (Figure 1D). These results indicate that ELA may be a potential prognostic marker for arrhythmia and AF.

The BNP was highly expressed in participants with AF (Figure 2A). To understand the role of ELA in predicting $\mathrm{AF}$ prognosis, bivariate correlation analysis was performed, and the results showed that the expression of ELA was negatively correlated with levels of BNP in all participants (Spearman $\mathrm{R}=-0.187, \mathrm{P}=0.033$ ) (Figure $2 B$ ). However, consistent with previous reports, TIMP-1 levels were positively correlated with BNP levels (Spearman $\mathrm{R}=0.2$, $\mathrm{P}=0.022$ ) (Figure 2C). Binary logistic regression analysis for AF indicated that ELA could be independently used as a prognostic biomarker for AF (95\% CI: 0.811-0.939, $\mathrm{P}<0.0001)$ (Table 2).

\section{Discussion}

The burden of heart arrhythmia is increasing with aging of the population, and $\mathrm{AF}$ is the most common type of heart arrhythmia (15). The presence of AF tends to exacerbate other heart complications such as HF, and the prognosis is often poor. Early diagnosis is beneficial for AF therapy and improves prognosis. At present, blood testing is one of the main diagnostic methods for identifying AF, which also helps to discover the underlying potencies of $\mathrm{AF}$ and heart damage. For example, BNP has been confirmed as a valuable predictor for the entire spectrum of HF disease severity (16). In this study, we characterized the baseline data of 131 patients with AF and PVST. The levels of NE, CK-MB, BUN, creatinine, uric acid, potassium, TB, DB, Fbg, HbA1c, and TT3 were high in participants with AF. The major risk factors of $\mathrm{AF}$ were age and high BNP levels. Furthermore, our findings revealed that ELA expression was low in patients with $\mathrm{AF}$ and those with high BNP levels 
Table 1 Baseline characteristics according AF as following

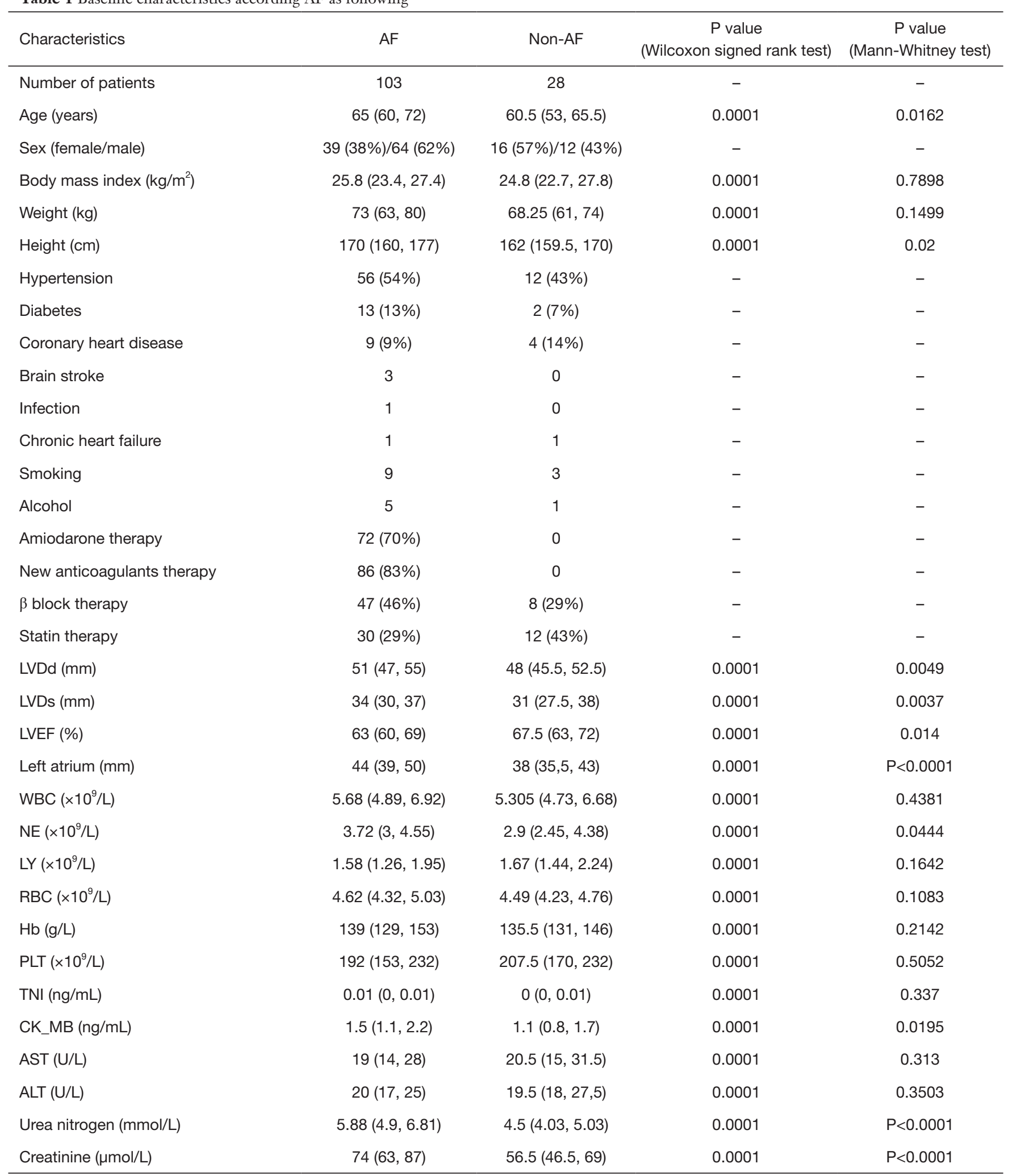

Table 1 (continued) 
Table 1 (continued)

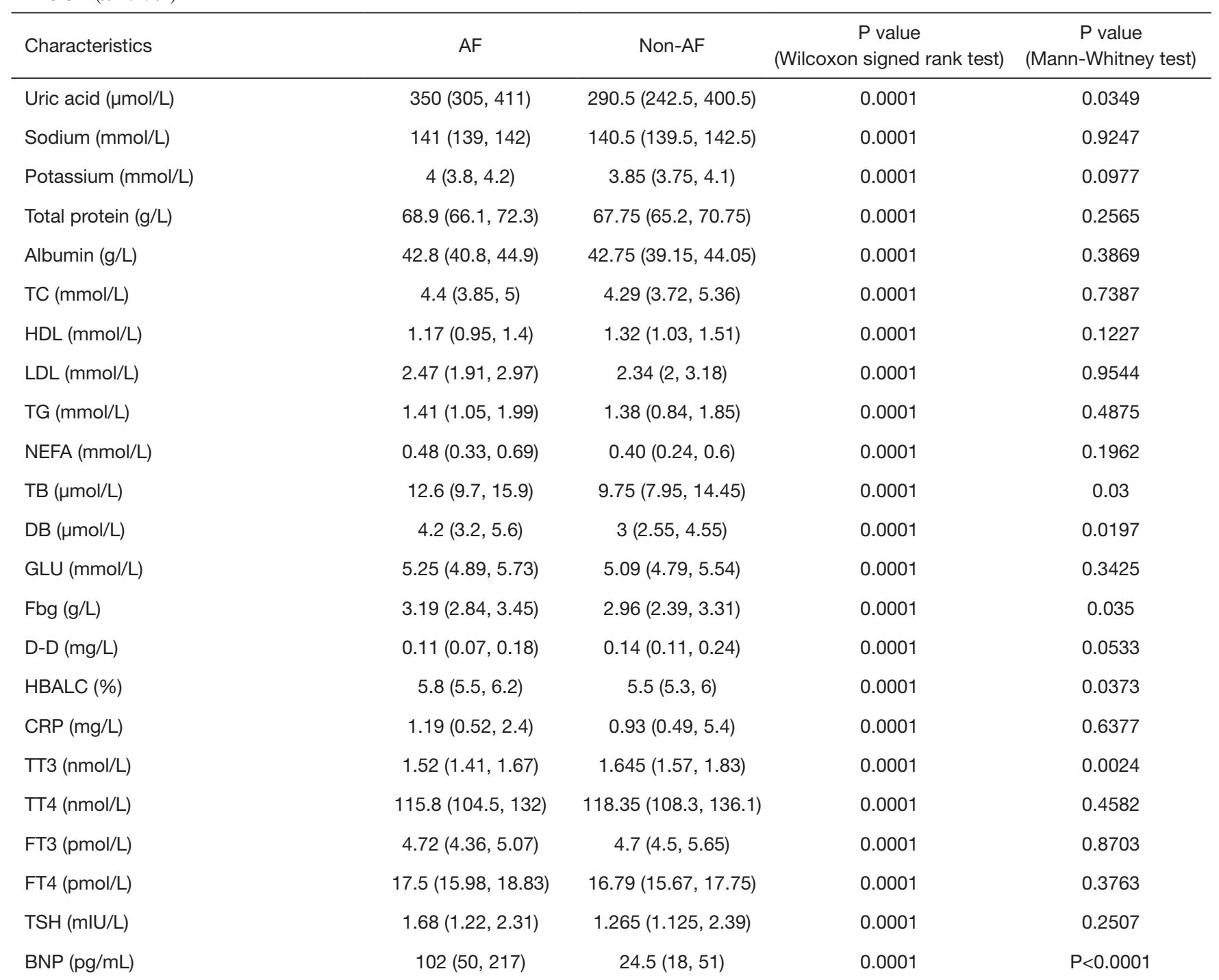

Data are shown as median (first quartile, third quartile) or $n$ (\%). AF, atrial fibrillation; LVDd, left ventricular end-diastolic diameter; LVDs, left ventricular end-systolic diameter; LVEF, left ventricular ejection fraction; WBC, white blood cell count; NE, neutrophil; LY, lymphocyte; RBC, red blood cells; Hb, hemoglobin; PLT, platelet count; TNI, troponin; CK-MB, creatine kinase MB; AST, aspartate aminotransferase; ALT, alanine transaminase; TC, total cholesterol; HDL, high density lipoprotein; LDL, low density lipoprotein; TG, triglyceride; NEFA, non-esterified fatty acid; TB, total bilirubin; DB, direct bilirubin; GLU, glucose; BNP, brain natriuretic peptide; Fbg, fibrinogen; D-D, d-dimer; HbA1c, glycosylated hemoglobin A1c; CRP, C-reactive protein; TT3, total triiodothyronine; TT4, total thyroxine; FT3, free triiodothyronine; FT4, free thyroxine; TSH, thyroid stimulating hormone.

$(>100 \mathrm{pg} / \mathrm{mL})$.

The circulating hormone ELA is required for heart development and plays an important role in cardiac development, angiogenesis, and cardiovascular physiology (14). It has been reported as downregulated in individuals with acute $\mathrm{HF}$ and its expression is low in isolated cases of AF (17). Herein, we studied the serum levels of ELA together with other biomarkers (BNP) in 103 patients with AF and 28 patients with PSVT, and found a low level of ELA in AF patients and in patients with high BNP expression ( $>100 \mathrm{pg} / \mathrm{mL})$. Furthermore, in blood samples, ELA expression was negatively correlated with BNP levels. As shown in Table 2, the binary logistic analysis showed that both ELA and BNP significantly contributed 
A

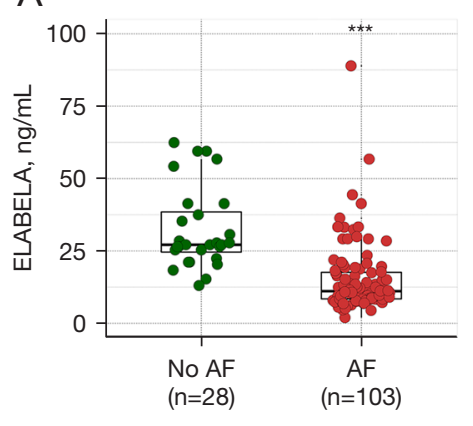

C

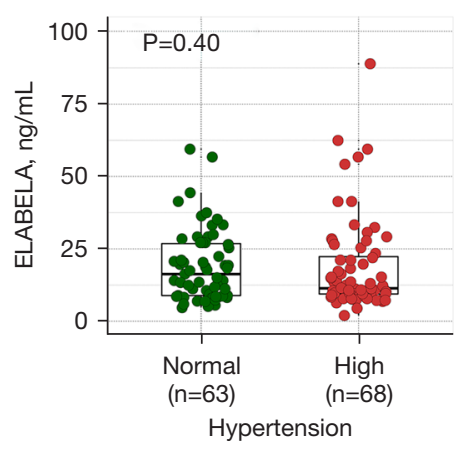

B

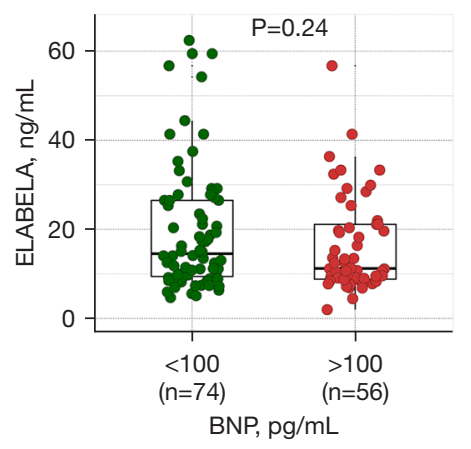

D

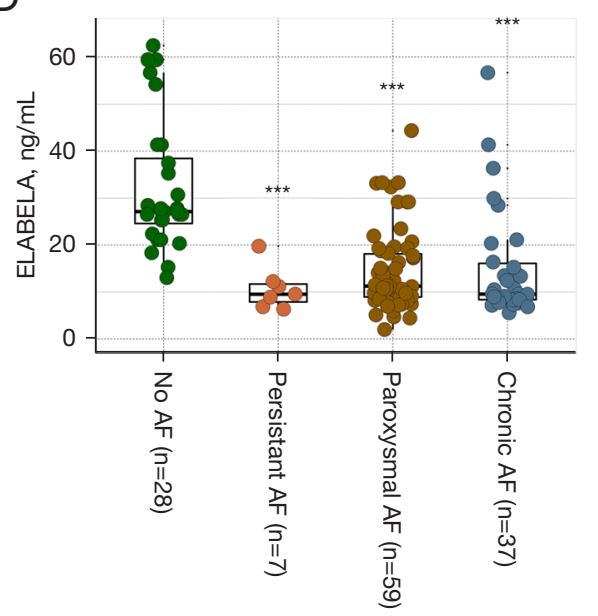

Figure 1 Low level of ELA expression in AF patients. (A) The ELA level was lower in AF patients than in PSVT patients $\left.{ }^{* * *} \mathrm{P}<0.001\right)$; (B) ELA expression was low in patients with increased BNP levels ( $>100 \mathrm{pg} / \mathrm{mL})$; (C) the ELA level was low in patients with hypertension; (D) ELA expression was low in patients with persistent $\mathrm{AF}$, paroxysmal $\mathrm{AF}$ and chronic $\mathrm{AF}$ compared with $\mathrm{PSVT}$ patients (*** $\mathrm{P}<0.001)$. AF, atrial fibrillation; BNP, brain natriuretic peptide; ELA, ELABELA; PSVT, paroxysmal supraventricular tachycardia.

to AF prognosis. Therefore, we hypothesized that ELA might be another potential biomarker for predicting $\mathrm{AF}$ and associated complications, such as HF.

In this study, we compared ELA expression in persistent $\mathrm{AF}$, paroxysmal AF, and chronic AF controlled with PVST patients, and ELA was expressed at low levels in all $3 \mathrm{AF}$ types. The ELA expression in participants with hypertension was lower than that in those without hypertension. These results suggest that ELA could protect the heart from risk factors, but the detailed molecular mechanism needs to be studied in greater detail.

In this study, we also evaluated the expressions of MMP-9 and TIMP-1 in patients. The levels of TIMP-1 were higher in participants with BNP levels of $>100 \mathrm{pg} / \mathrm{mL}$ and MMP9 levels were higher in AF patients controlled with PVST, although the difference was not significant. The expression of TIMP-1 was high in persistent $\mathrm{AF}$ and chronic $\mathrm{AF}$, but not in paroxysmal AF. Consistently, MMP-9 and TIMP1 have been reported to contribute to extracellular matrix remodeling of atria in $\mathrm{AF}$ (13). In this study, we did not find obvious correlation between expression of MMP-9, TIMP1 and ELA. Maybe more samples should be provided to further identified this point. In all participants, TIMP1 expression positively correlated with BNP expression. Therefore, these risk factors may be used as auxiliary risk factors for different types of AF prognosis. So, ELA could be used as predictive biomarker for AF and maybe benefit for preventing $\mathrm{AF}$ complications.

\section{Conclusions}

High levels of BNP are markers of impaired cardiac 


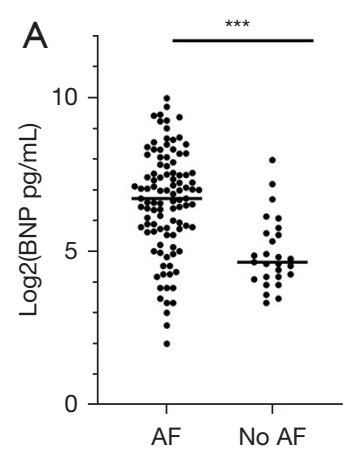

B

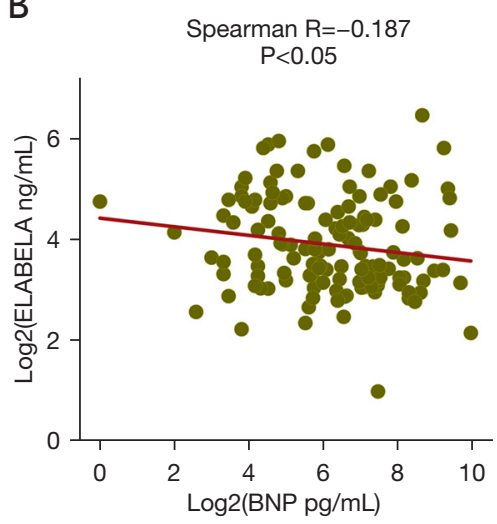

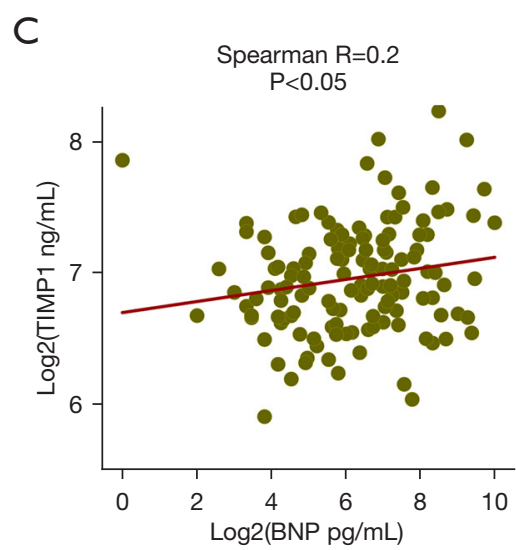

Figure 2 The ELA level was negatively correlated with BNP. (A) The BNP level was higher in patients with AF than in patients with PSVT $\left({ }^{* * *} \mathrm{P}<0.001\right)$; (B) the bivariate correlation analysis showed that that ELA level was negatively correlated with the BNP level (Spearman $\mathrm{R}=-0.187, \mathrm{P}=0.033$ ); (C) the bivariate correlation analysis showed that TIMP-1 was positively correlated with the BNP level (Spearman $\mathrm{R}=0.2, \mathrm{P}=0.022$ ). $\mathrm{AF}$, atrial fibrillation; BNP, brain natriuretic peptide; ELA, ELABELA; TIMP-1, tissue inhibitor of metalloproteinase 1; PSVT, paroxysmal supraventricular tachycardia.

Table 2 Binary logistic analysis for AF

\begin{tabular}{llc}
\hline Variable & $95 \% \mathrm{Cl}$ & $\mathrm{P}$ value \\
\hline Age & $0.158-7.230$ & 0.945 \\
BMI & $0.896-1.246$ & 0.511 \\
Left atrium & $0.899-1.309$ & 0.398 \\
LVD & $0.754-1.512$ & 0.712 \\
LVS & $0.606-1.331$ & 0.592 \\
IVS & $0.583-1.408$ & 0.661 \\
LVEF & $0.918-1.074$ & 0.864 \\
CK-MB & $0.639-2.915$ & 0.422 \\
MMP9 & $0.999-1.004$ & 0.233 \\
TIMP1 & $0.951-1.002$ & 0.076 \\
ELABELA & $0.811-0.939$ & 0.000 \\
BNP & $1.014-1.047$ & 0.000 \\
\hline
\end{tabular}

$\mathrm{Cl}$, confidence interval; AF, atrial fibrillation; BMI, body mass index; LVDd, left ventricular end-diastolic diameter; LVDs, left ventricular end-systolic diameter; LVEF, left ventricular ejection fraction; CK CK-MB, creatine kinase MB; MMP-9, matrix metalloproteinase-9; TIMP-1, tissue inhibitor of metalloproteinase 1. 
function and associated complications of $\mathrm{HF}$ and stroke. The ELA level was much lower in patients with impaired cardiac function, indicating its protective role in the cardiovascular system. In conjunction with BNP and other high-risk factors of cardiac insufficiency, ELA could be used as a prognostic biomarker for patients with arrhythmia.

\section{Acknowledgments}

Funding: This work was supported by National Natural Scientific Foundation of China Grants (No. 81900373), Outstanding Youth Training Funds of Shanghai Renji Hospital (PYIII-17-034), China International Medical Fund (z-2016-23-2101018), and China International Medical Foundation (Z-2019-42-1908-2).

\section{Footnote}

Reporting Checklist: The authors have completed the MDAR checklist. Available at https://dx.doi.org/10.21037/ jtd-21-1728

Data Sharing Statement: Available at https://dx.doi. org/10.21037/jtd-21-1728

Conflicts of Interest: All authors have completed the ICMJE uniform disclosure form (available at https://dx.doi. org/10.21037/jtd-21-1728). The authors have no conflicts of interest to declare.

Ethical Statement: The authors are accountable for all aspects of the work in ensuring that questions related to the accuracy or integrity of any part of the work are appropriately investigated and resolved. The study was conducted with the approval of the Ethics Committee of Renji Hospital, School of Medicine, Shanghai Jiao Tong University (approval No. KY2021-131-B) and in accordance with the Declaration of Helsinki (as revised in 2013). Samples were collected and analyzed after the provision of written informed consent.

Open Access Statement: This is an Open Access article distributed in accordance with the Creative Commons Attribution-NonCommercial-NoDerivs 4.0 International License (CC BY-NC-ND 4.0), which permits the noncommercial replication and distribution of the article with the strict proviso that no changes or edits are made and the original work is properly cited (including links to both the formal publication through the relevant DOI and the license). See: https://creativecommons.org/licenses/by-nc-nd/4.0/.

\section{References}

1. Lindberg T, Wimo A, Elmståhl S, et al. Prevalence and Incidence of Atrial Fibrillation and Other Arrhythmias in the General Older Population: Findings From the Swedish National Study on Aging and Care. Gerontol Geriatr Med 2019;5:2333721419859687.

2. Moreira LM, Takawale A, Hulsurkar M, et al. Paracrine signalling by cardiac calcitonin controls atrial fibrogenesis and arrhythmia. Nature 2020;587:460-5.

3. Inoue T, Suematsu Y. Poor enhancement pattern of left atrial appendage in cardiac computed tomography is associated with stroke in persistent atrial fibrillation patients. J Thorac Dis 2019;11:3315-24.

4. Bose A, Truong QA, Singh JP. Biomarkers in electrophysiology: role in arrhythmias and resynchronization therapy. J Interv Card Electrophysiol 2015;43:31-44.

5. NakanoY, Niida S, Dote K, et al. Matrix metalloproteinase-9 contributes to human atrial remodeling during atrial fibrillation. J Am Coll Cardiol 2004;43:818-25.

6. Ho L, Tan SY, Wee S, et al. ELABELA Is an Endogenous Growth Factor that Sustains hESC Self-Renewal via the PI3K/AKT Pathway. Cell Stem Cell 2015;17:435-47.

7. Zeng XX, Wilm TP, Sepich DS, et al. Apelin and its receptor control heart field formation during zebrafish gastrulation. Dev Cell 2007;12:391-402.

8. Ho L, van Dijk M, Chye STJ, et al. ELABELA deficiency promotes preeclampsia and cardiovascular malformations in mice. Science 2017;357:707-13.

9. Wang J, Zhou Y, Wang Q, et al. Elabela: A Novel Biomarker for Right Ventricular Pressure Overload in Children With Pulmonary Stenosis or Pulmonary Atresia With Intact Ventricular Septum. Front Cardiovasc Med 2020;7:581848.

10. Sato T, Sato C, Kadowaki A, et al. ELABELA-APJ axis protects from pressure overload heart failure and angiotensin II-induced cardiac damage. Cardiovasc Res 2017;113:760-9.

11. Ma Z, Zhao L, Zhang YP, et al. Declined ELABELA plasma levels in hypertension patients with atrial fibrillation: a case control study. BMC Cardiovasc Disord. 2021;21:390.

12. Uziębło-Życzkowska B, Krzesiński P, Jurek A, et al. Left 
Ventricular Ejection Fraction Is Associated with the Risk of Thrombus in the Left Atrial Appendage in Patients with Atrial Fibrillation. Cardiovasc Ther 2020;2020:3501749.

13. Chen CL, Huang SK, Lin JL, et al. Upregulation of matrix metalloproteinase- 9 and tissue inhibitors of metalloproteinases in rapid atrial pacing-induced atrial fibrillation. J Mol Cell Cardiol 2008;45:742-53.

14. Kim YM, Lakin R, Zhang H, et al. Apelin increases atrial conduction velocity, refractoriness, and prevents

Cite this article as: Cui C, Zhou H, Xu J. ELABELA acts as a protective biomarker in patients with atrial fibrillation. J Thorac Dis 2021;13(12):6876-6884. doi: 10.21037/jtd-21-1728 inducibility of atrial fibrillation. JCI Insight 2020;5:126525.

15. Lip GY, Fauchier L, Freedman SB, et al. Atrial fibrillation. Nat Rev Dis Primers 2016;2:16016.

16. Yoo BS. Clinical Significance of B-type Natriuretic Peptide in Heart Failure. J Lifestyle Med 2014;4:34-8.

17. Ellinor PT, Low AF, Macrae CA. Reduced apelin levels in lone atrial fibrillation. Eur Heart J 2006;27:222-6.

(English Language Editor: J. Jones) 
A
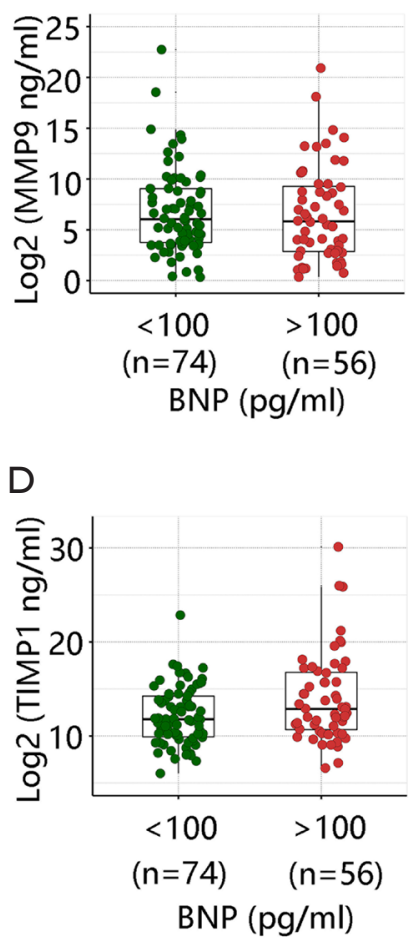

G

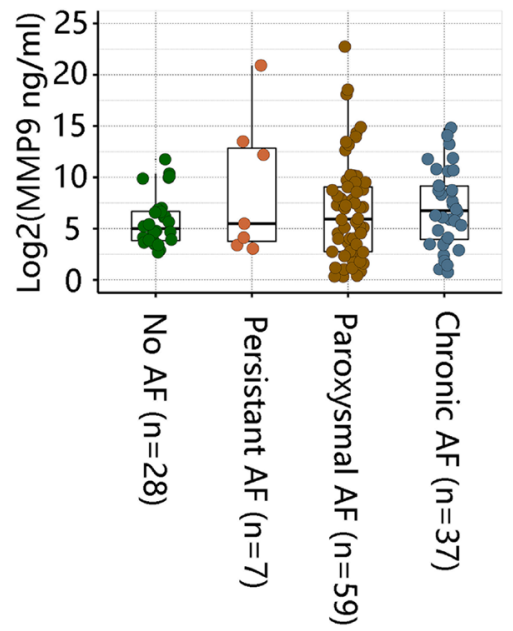

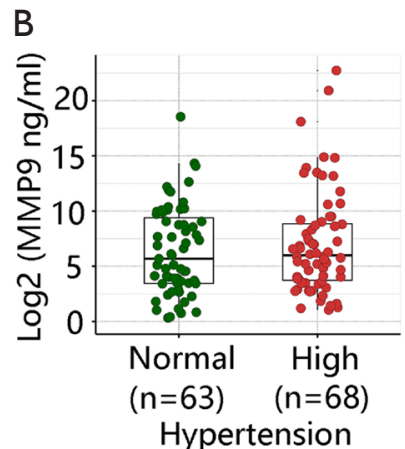
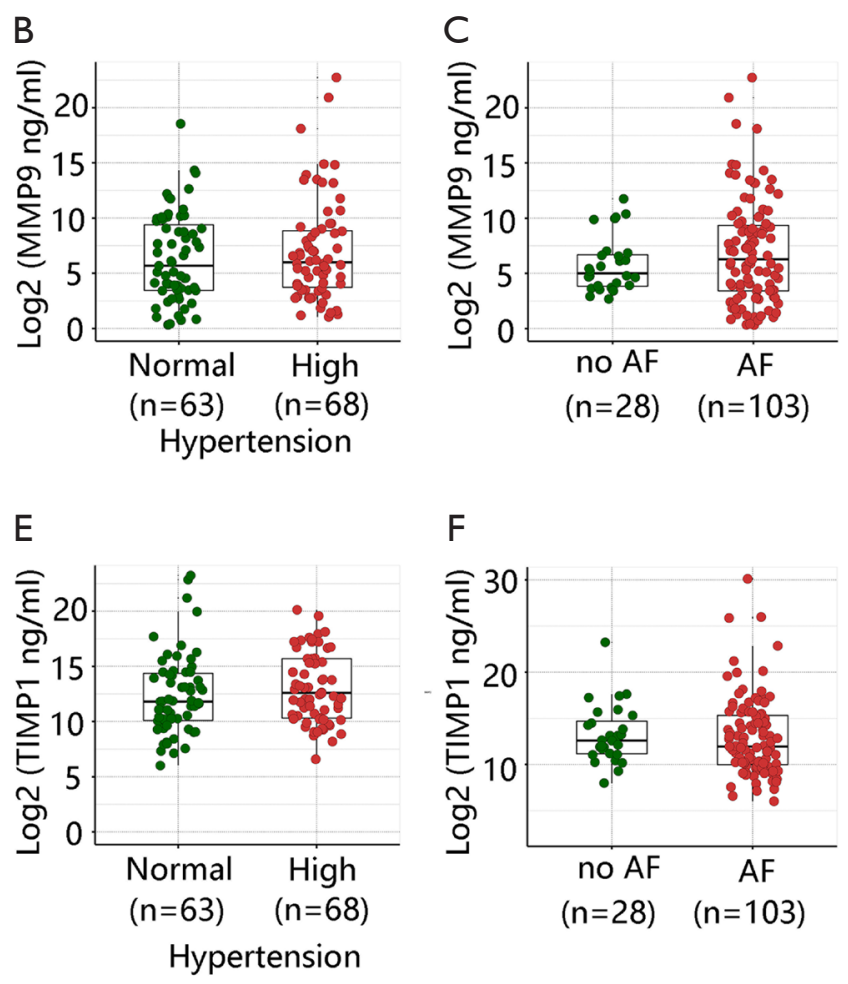

$\mathrm{H}$

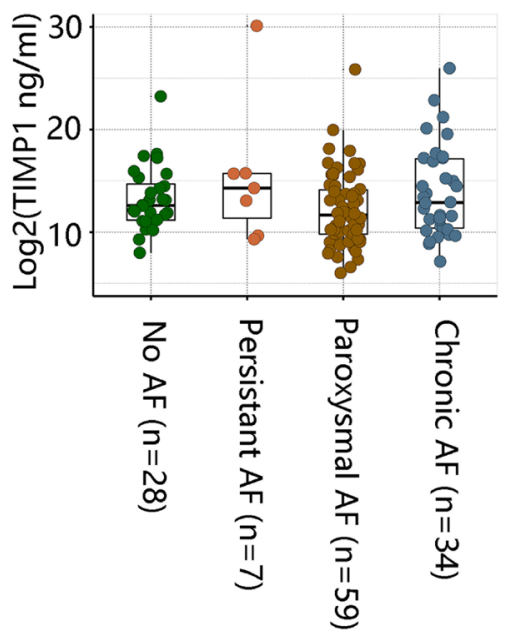

Figure S1 Level of MMP-9 and TIMP-1 in patients with high risk factor. (A) Level of MMP-9 showed no difference in patients with different levels of BNP; (B) level of MMP-9 showed no difference in patients with hypertension or not; (C) MMP-9 level was higher in AF patients compared with PSVT control; (D) level of TIMP-1 showed higher level in patients with increased BNP level (>100 pg/mL); (E) level of TIMP-1 showed higher level in patients with hypertension; (F) TIMP-1 expression showed lower level in AF patients; (G) MMP9 expression showed higher level in all kinds of AF compared with PSVT control; (H) TIMP-1 expression showed higher level in persistent $\mathrm{AF}$ and Chronic AF, but not in paroxysmal AF. AF, atrial fibrillation; BNP, brain natriuretic peptide; ELA, ELABELA; TIMP-1, tissue inhibitor of metalloproteinase 1; PSVT, paroxysmal supraventricular tachycardia; MMP-9, matrix metalloproteinase-9. 